\title{
Audiovestibular Function Deficits in Vestibular Schwannoma
}

\author{
Constantin von Kirschbaum and Robert Gürkov \\ Department of Otorhinolaryngology and Head and Neck Surgery, Grosshadern Medical Center, University of Munich, \\ Marchioninistr. 15, 81377 Munich, Germany
}

Correspondence should be addressed to Robert Gürkov; robert.guerkov@med.uni-muenchen.de

Received 30 June 2016; Revised 12 August 2016; Accepted 24 August 2016

Academic Editor: Stefan Rampp

Copyright (c) 2016 C. von Kirschbaum and R. Gürkov. This is an open access article distributed under the Creative Commons Attribution License, which permits unrestricted use, distribution, and reproduction in any medium, provided the original work is properly cited.

\begin{abstract}
Introduction. Vestibular schwannomas (VS) are benign tumours of the vestibular nerve and can lead to hearing loss, tinnitus, vertigo, facial palsy, and brainstem compression. Audiovestibular diagnostic tests are essential for detection and treatment planning. Methods. Medline was used to perform a systematic literature review with regard to how audiovestibular test parameters correlate with symptoms, tumour size, and tumour location. Results. The auditory brainstem response can be used to diagnose retrocochlear lesions caused by VS. Since hearing loss correlates poorly with tumour size, a retrocochlear lesion is probably not the only cause for hearing loss. Also cochlear mechanisms seem to play a role. This can be revealed by abnormal otoacoustic emissions, despite normal ABR and new MRI techniques which have demonstrated endolymphatic hydrops of the inner ear. Caloric and head impulse tests show frequency specific dynamics and vestibular evoked myogenic potentials may help to identify the location of the tumour regarding the involved nerve parts. Conclusion. In order to preserve audiovestibular function in VS, it is important to stop the growth of the tumour and to avoid degenerative changes in the inner ear. A detailed neurotological workup helps to diagnose VS of all sizes and can also provide useful prognostic information.
\end{abstract}

\section{Introduction}

Vestibular schwannoma (VS) can lead to sensorineural hearing loss, tinnitus, and vertigo. Patients with asymmetric hearing loss, unilateral tinnitus, or vertigo should be evaluated for the presence of a VS.

VS are benign tumours arising from the sheath of the vestibular nerve. They cause dysfunction of the nerves by compressing the nerve fibre and obstructing the blood supply to the nerves $[1,2]$. VS may present with a variety of clinical features. Hearing loss is the most common first symptom while vertigo is often the most distressing symptom for the patient. However, hearing loss may also be absent in case of an extrametal growth pattern.

The estimated annual incidence of these tumours ranges from approximately 0.6 to 1.9 per 100,000 [3].

Currently there exist several classifications of VS, based on the tumour size and location. Table 1 gives a summary of the two most common classifications according to Koos and Samii.

Audiovestibular function tests, such as pure tone audiometry (PTA), otoacoustic emissions (OAEs), auditory brainstem response $(\mathrm{ABR})$, caloric irrigation, head impulse test (HIT), and vestibular evoked myogenic potentials (cVEMPs and oVEMPs) are important for initial diagnosis as well as subsequent monitoring of disease progression.

The current gold standard of diagnosis is MR imaging, but because MRI scans are expensive, they are not universally utilized to screen all patients with audiovestibular symptoms [4]. Thus, VS may be misdiagnosed as a peripheral disease, such as noise-induced hearing loss, presbyacusis, tinnitus, vestibulopathy, Menière's disease, or sudden deafness with vertigo.

Furthermore, precise preoperative audiovestibular assessment can have implications on the decision of the therapeutic approach such as wait and scan, radiotherapy, or microsurgery.

\section{Methods}

English and German articles published between 1972 and 2016 and available on Medline were reviewed in order to produce a summary of the current knowledge with regard 
Table 1: Tumour classification according to Koos et al. [24] and Samii and Matthies [25].

\begin{tabular}{lcc}
\hline Grade (Koos et al.) & Grade (Samii and Matthies) & Definition of tumour size \\
\hline I & T1 & Purely intracanalicular lesion \\
II & T2 & VS protruding into the cerebellopontine angle without brainstem contact \\
IIa & T2 & Tumour diameter $<1 \mathrm{~cm}$ \\
IIB & T2 & Tumour diameter $1-1,8 \mathrm{~cm}$ \\
& T3a & Filling cerebellopontine angle cistern \\
III & T3b & Reaching the brainstem \\
& T4a & Brain stem compression \\
IV & T4b & Severely dislocating the brainstem and compressing the fourth ventricle \\
\hline
\end{tabular}

to audiovestibular functional changes in patients with VS as diagnosed by MR imaging.

\section{Results}

\subsection{Audiological Function Tests}

3.1.1. Vestibular Schwannomas and Audiometry. Sudden sensorineural hearing loss may be an initial symptom of a VS [5]; therefore, audiometry has been regarded as an initial step in the diagnostic workup for VS.

It has been shown that VS has been diagnosed in 0.8$47.5 \%$ of patients with sudden sensorineural hearing loss $[6,7]$.

To investigate predictive factors or symptoms of future hearing loss Jethanamest et al. retrospectively reviewed patients between 2002 and 2013 who chose observation for their initial management. The estimated median time to loss of serviceable hearing was 6.3 years. No symptoms or factors were identified to be predictive of future hearing loss. However, symptoms of disequilibrium or imbalance appeared to be associated with an increased risk of subsequent tumour growth [8].

Wagner et al. found that the degree of hearing loss before treatment is significantly influenced by the age of the patient $(p<0.001)$ but not by tumour size. At follow-up examinations after treatment with microsurgery and radiotherapy, hearing was significantly worse in those patients with a large VS. Age was not a significant determinant for loss of hearing on the follow-up visits $(p<0.08)$ [9].

Another study noted that $45 \%$ of those patients who initially had a hearing ability of AAO class A on the tumour affected side lost class A hearing after 5 years [10].

Concerning speech discrimination, Stangerup et al. demonstrated during a 33 -year period that $59 \%$ of the patients with a speech discrimination better than $70 \%$ preserved good hearing after a mean of 4.7 years of observation. $69 \%$ of the patients with a speech discrimination score of $100 \%$ at diagnosis maintained good hearing after more than 10 years. Of the patients with only a small discrimination loss at diagnosis, 38\% maintained good hearing [11].

Tumour growth less than or equal to 2.5 millimeters per year has been shown to lead to higher rates of hearing preservation. For patients with a small tumour and normal speech discrimination, the main indication for active treatment should be the established tumour growth [11, 12].
Day et al. found in a total of 44 patients that normal hearing or low-frequency hearing loss was correlated with small tumour size. Those with mid- or high-frequency hearing loss had a medium-sized tumour, while those with global frequency hearing loss or total deafness had tumour size larger than $2.5 \mathrm{~cm}$. The authors explained the hearing loss by tumour compression of the cochlear nerve, since most VS originate from the vestibular nerve [13]. This association of small tumour size with low-frequency hearing loss may suggest the presence of endolymphatic hydrops in some of these patients, since hydrops is typically associated with lowfrequency hearing loss.

Concerning primary inner ear schwannomas (PIES), that is, schwannomas arising from the labyrinth, a systematic review of 243 patients diagnosed from 1933 to 2011 showed that unilateral hearing loss was the most frequent presenting symptom (99\%). Vertigo and abnormal balance were more common among tumours involving the vestibular system $(p<0.01)$ [14]. Positional vestibular symptoms and mixed hearing loss occur especially in intralabyrinthine schwannomas and are suggested to result from the tumour's mass effect within the labyrinth [15]. Especially in intralabyrinthine schwannoma a diagnostic delay of up to 15 years has been reported. Since radiographic findings in MRI may be very subtle, intralabyrinthine schwannomas can easily be overlooked, so that they are frequently diagnosed only years after the onset of symptoms [16].

Overall, there is limited data on the long-term auditory symptoms in patients with sporadic small- and medium-sized VS. The initial treatment strategy for VS is being discussed controversially. The overall prognosis for hearing in sporadic VS is poor regardless of treatment strategy. Good baseline hearing proved to be a strong predictor for maintained serviceable hearing. Observation was associated with the highest rate of hearing preservation [17], although this might be due to a selection bias.

3.1.2. Vestibular Schwannomas and ABR. After audiometry, the next diagnostic test of choice for patients with clinical suspicion of a VS (unilateral or asymmetrical sensorineural hearing loss) was the ABR. However, with improvements in MRI diagnostics in the late 1980s, the usefulness of the ABR in the diagnostic workup of VS has diminished and MRI is nowadays widely considered to be the gold standard for diagnosing retrocochlear pathology. 
Currently, the role of the ABR remains controversial. The criteria for assessing abnormal responses on ABR tests have varied considerably among studies. Some authors argue that the ABR has no place in today's management and that MRI should be the initial screening test when there is a suspicion of VS [18]. Other authors argue that there is still a place for the ABR in the diagnostic algorithm due to the high cost and lower availability of MRI [19].

The ABR has a high dependency on the tumour size and the diagnostic sensitivity is especially low for smaller tumours [20].

This was also shown in a study by Koos et al. in which multiple online databases were assessed in order to evaluate the usefulness of ABR tests in 3314 patients from 1978 to 2009. The inclusion criterion was the presence of a surgically or radiographically confirmed VS. The pooled sensitivity of $\mathrm{ABR}$ was $93.4 \%, 85.8 \%$ for tumours $<1 \mathrm{~cm}$, and $95.6 \%$ for tumours $>1 \mathrm{~cm}$. Moreover, the sensitivity of ABR was higher for extracanalicular than for intracanalicular tumours. The authors concluded that for patients with lower clinical suspicion of a VS an ABR can still provide valuable additional information on which the decision to obtain an MRI can be based.

However, a small number of tumours with audiometrically documented hearing loss demonstrate a normal ABR [21], which supports the hypothesis that schwannomas can also cause hearing loss by purely cochlear mechanisms.

\subsubsection{Vestibular Schwannomas and Otoacoustic Emissions.}

OAEs are active mechanical responses from the cochlea which provide information about the integrity of the preneural cochlear receptor mechanisms. Clinical experience has shown that OAEs are often absent or compromised in VS. In order to objectify the effects of retrocochlear disease on distortion-product otoacoustic emissions (DPOAEs) Telishi et al. classified the DPOAE patterns as cochlear or noncochlear. In a large series of patients with unilateral VS they showed that the majority of ears with tumours demonstrated cochlear (57\%), rather than noncochlear (41\%) patterns of DPOAEs [22].

The occurrence of cochlear pathology has implications regarding the accuracy of OAEs as a diagnostic tool for VS. If one assumes that VS affect the retrocochlear function (hearing impairment is purely neural in origin) and have little effect on the cochlea itself then VS patients should have preserved OAEs. However, it seems that amplitudes of DPOAEs begin to decrease already at the early stages of hearing loss in VS patients. Comparing a group of VS patients with normal/symmetrical hearing and a group of VS patients with mild hearing loss (threshold at any tested frequency better than $45 \mathrm{~dB}$ ) on the tumour ear side, Gouveris et al. showed that DPOAE amplitudes do not differ strongly between the ears in VS patients with normal/symmetrical hearing. But DPOAEs are decreased compared with the nontumour ear in VS patients with even mild hearing loss. The tumour size did not differ significantly between these two groups, which suggests a cochlear origin of early hearing loss in these patients. The authors conclude that DPOAEs may be used in a clinical setting to monitor progression of cochlear damage at the early stages of hearing impairment in VS patients [23].

\subsection{Vestibular Function Tests}

3.2.1. Vestibular Schwannomas and Caloric Response. Vertigo has been reported to be one of the risk factors for the growth of VS [26]. Therefore, vestibular function tests such as the caloric test should be used routinely in the workup of VS. By using videonystagmography (VNG) or electronystagmography (ENG), caloric responses can be observed and quantified in terms of slow-phase nystagmus velocities generated during warm and cold irrigations of each ear. The asymmetry between the two horizontal semicircular canals is usually calculated by the Jongkees formula. When unilateral weakness (UW) is less than $25 \%$, the caloric response is regarded as normal.

With the caloric test it is possible to unilaterally stimulate the horizontal semicircular canal, which is innervated by the superior part of the vestibular nerve. Thus, one might think that the caloric response is only significant when the superior branch of the vestibular nerve is affected by the VS. Borgmann et al. tested whether ENG results could predict the nerve of origin before surgery. They defined pathologic caloric test findings as an indicator for superior vestibular nerve schwannomas (SVN) involvement and a normal caloric response as a sign of inferior vestibular nerve schwannomas (IVN) origin. As a result, the nerve of origin could be predicted in 90 of 111 patients (81\%), which means that pathologic results in preoperative ENG were significantly more frequent in patients with SVN schwannomas. They concluded that the caloric test helps to predict the nerve of origin of a VS and can be used indirectly as a prognostic factor for hearing preservation because hearing loss due to surgery was significantly lower in patients with tumours of the SVN [27].

In contrast Ushio et al. demonstrated in a study of 109 VS patients that the percentage of abnormal responses in caloric tests was not different between patients with SVN and those with IVN [2]. These results showed no clear correlation between the results of caloric tests and the nerve origin of the tumour.

Tringali et al. observed a good correlation between caloric weakness and tumour size when they tested the preoperative response of 629 tumour patients. In the group of patients with UW $>70 \%$, who had a larger tumour size, postoperative facial palsy was more frequent. Postoperative hearing preservation was more frequently observed in the "normal group" with a UW $<20 \%$. They concluded that a normal caloric response can be a good predictive factor for hearing preservation and normal postoperative facial function [28].

In a prospective pilot study on patients with VS, Wagner et al. compared groups with VS $<20 \mathrm{~mm}$ and $\geq 20 \mathrm{~mm}$. In group 1, the median loss of vestibular function was $+10.5 \%$; in group 2 (with a tumour size $\geq 20 \mathrm{~mm}$ ) a higher degree of loss of vestibular function (median UW 36\%) was found. Treatment by micro- and radiosurgery caused a further decrease of vestibular function in both groups. So vestibular function clearly correlated with the size of the tumour and 
also with the method of treatment used. The majority of patients complained about vertigo before and after treatment [9].

Ushio et al. also found that the mean tumour size in patients showing abnormal responses in caloric testing was larger than that in patients showing normal responses. This tendency was not observed for patients with VS within the internal acoustic canal [2]. The same observation was made by Suzuki in patients with unilateral VS histologically diagnosed by surgery. There was no significant difference between patients with normal caloric responses and those with canal paresis with respect to the size of the tumour measured for intracanalicular tumours [29].

On the contrary, the results of a study by Teggi et al. showed that vestibular function is influenced by intracanalicular length and diameter of the tumour, rather than by total tumour volume. The subgroup of 30 patients with a greater intracanalicular length of the tumour presented a higher value of UW than the subgroup with a smaller length [30].

In summary, the available data suggest that the severity of the UW correlates with degree of infiltration of the cochleovestibular nerve by the tumour. This means that an abnormal caloric response is a prognostic factor for hearing preservation surgery [31].

\subsubsection{Vestibular Schwannomas and Head Impulse Test. Both} the caloric irrigation and the head impulse test (HIT) serve to evaluate the horizontal vestibuloocular reflex (VOR). The HIT applies high-acceleration small-amplitude head impulses around an earth-vertical axis while the patient is fixating a stationary target. A catch-up saccade is observed if the eye no longer compensates for the head movement and accordingly this test is classified as pathologic. The probability of a pathologic HIT generally increases with increasing UW of caloric examination. For patients with a UW within normal limits, a pathological HIT is very unlikely [32].

At present the HIT is the only bedside examination that enables the identification of the side of a unilateral hypofunction of the peripheral vestibular system.

However, a VOR deficit may not be diagnosed because corrective saccades cannot always be detected by simple observation. Besides the manual HIT as described by Halmagyi et al. [33], which can be performed quickly as a bedside test, there is the video head impulse test (vHIT). This allows the identification not only of overt saccades but also of covert saccades occurring during the head movement which are not visible to the naked eye.

2013 Blödow et al. examined 142 patients with acute or chronic vestibular syndrome and found that $47.6 \%$ had a pathological vHIT whereas $52.4 \%$ had a normal test result. Covert catch-up saccades could be demonstrated in $13.7 \%$ whereas in $86.3 \%$ overt catch-up saccades alone or in combination with covert catch-up saccades were found. The authors concluded that the vHIT is superior to the clinical HIT because in approximately one in eight cases with acute or chronic peripheral vestibular syndrome a covert catch-up saccade can be detected, which would otherwise have been undetectable by the clinical HIT [34].
The same group recently examined 46 patients with VS and showed that caloric irrigation exhibits a higher sensitivity than HIT (72\% versus $41 \%$ ) and both tests show only a moderate correlation. Tumour size and hearing level were significantly correlated with caloric abnormalities but not with HIT findings [35].

The finding of a selective impairment of the VOR in the low-frequency range (caloric) was also shown in a case of an intralabyrinthine schwannoma, whereas the high-frequency VOR (HIT) remains intact [36]. This constellation is typical for Menière's disease.

However, neither study could find an explanation for the higher sensitivity of the caloric test. Both tests measure the VOR but at different temporal frequencies: the HIT with short head impulses tests high frequencies up to $5 \mathrm{~Hz}$ [37] and the caloric irrigation tests lower frequencies around $0.003 \mathrm{~Hz}$ [33].

Vestibular testing at different frequencies provides deeper insights into VOR function and can help in detecting cerebellopontine lesions. According to the current literature, both tests have to be considered complementary and are valuable for both diagnostic and therapeutic decisions.

The HIT and the bithermal caloric irrigation are different in terms of not only temporal frequency, but also the method of stimulation. The HIT causes a physiologic endolymphatic flow as a consequence of rapid head impulses. In contrast, caloric irrigation induces endolymphatic flow due to a temperature gradient from one side of the canal to the other. Caloric irrigation additionally stimulates the inner ear in a non-gravity-dependent way [38].

It still remains unclear whether the dissociation of the caloric test and the HIT in patients with VS is due to the varying temporal frequencies or the differing methods of stimulation. Of note, the predilection for caloric test abnormality is typical for patients with Menière's disease (as opposed to, e.g., vestibular neuritis which typically affects both tests) and has been shown to correlate with the degree of endolymphatic hydrops herniation into the horizontal semicircular canal. Therefore, this feature might suggest endolymphatic hydrops in some of these patients.

3.2.3. Vestibular Schwannomas and VEMPs. VEMPs provide information on otolith organ function. The conventional method for recording VEMPs involves measuring electromyographic activity from surface electrodes placed over the tonically activated sternocleidomastoid muscles. The cervical VEMP (cVEMP) is a manifestation of the vestibulocollic reflex [39]. VEMPs can also be recorded from the extraocular muscles using surface electrodes placed near the eyes. These ocular VEMPs (oVEMPs) are a manifestation of the vestibuloocular reflex [40]. Stimulation of the vestibular system is possible using air-conducted sound (ACS) and bone-conducted vibration (BCV).

In recent years the role of cVEMPs and oVEMPs in the assessment of patients with VS has gained increased attention. A number of studies have demonstrated that VEMPs have an important clinical value in the diagnosis of VS because sometimes an abnormal VEMP result may be the 
only sign of a unilateral VS (the caloric and hearing test being normal) [41].

Physiological and clinical studies have shown that cVEMPs to ACS mainly reflect the function of saccular afferents and the inferior vestibular pathway [42-44].

On the contrary, clinical studies in patients with unilateral VS [45, 46] and animal studies using guinea pigs [47] have suggested that oVEMPs to BCV are likely to reflect the function of the utricle and superior vestibular pathway.

Recently VEMPs have been applied especially for classifying VS according to the involved nerves prior to surgery or radiotherapy.

Iwasaki et al. postulated that BCV oVEMPs mainly reflect the function of the SVN. They hypothesised that if the oVEMPs to BCV mainly reflect the function of the utricular afferents, the results should mostly coincide with those of the caloric test rather than with cVEMPs to ACS. Among ten patients with inconsistent results in these three vestibular tests, seven (70\%) showed corresponding results between oVEMPs to BCV and the caloric test, whereas only one patient (10\%) showed correspondence between oVEMPs to BCV and cVEMPs to ACS [46], thus supporting the authors' hypothesis.

In a bigger cohort of 45 patients with untreated unilateral VS, the results of oVEMPs to ACS had a significant correlation with those of oVEMPs to BCV. These findings support the hypothesis that oVEMPs in response to both ACS and $\mathrm{BCV}$ are predominantly mediated by the superior vestibular nerve and probably both reflect the function of the utricle [48].

However, according to other studies the nerve of origin of tumours cannot be predicted based on VEMPs. In a study of 130 patients histologically diagnosed by surgery, VEMPs in patients with tumours arising from the SVN were not significantly different from those in patients with tumours of the IVN [29]. Ushio et al. also could not find a clear correlation of VEMP results with the nerve origin of the tumour. The percentage of patients showing abnormal responses was not different between 37 patients with VS of the SVN and 26 patients with VS of the IVN. Also, no difference was observed for patients with VS within the internal acoustic canal. The authors hypothesised that large VS affect functions of both the SVN and the IVN, irrespective of the nerve origin, because the space of the internal acoustic canal is limited [2]. As a result tumours injure or compress both parts of the vestibular nerve as they grow. Complicating matters even further, it is likely that these efforts to predict the affected nerve can also be obscured by diffuse labyrinthine damage.

With regard to tumour size, several authors describe that, in VS, ACS cVEMPs are absent or decreased in amplitude in up to $80 \%$ of cases [49-51]. The amplitude decreases in association with an increase in tumour size. Larger tumours and those located more medially are more commonly associated with cVEMP abnormalities [13, 52].

Tumour compression of the brainstem and the vestibular spinal tract and compression of the myelin sheath of the IVN, resulting in demyelination of the nerve, were also correlated with cVEMP latencies $[29,46]$.

However, comparing the tumour size within the internal acoustic canal, Ushio et al. could not observe a difference

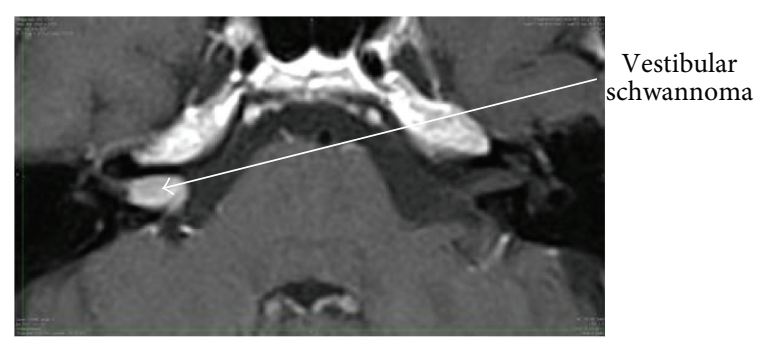

FIGURE 1: A vestibular schwannoma shown in a contrast-enhanced T1-weighted sequence.

between patients showing abnormal VEMP responses and patients showing normal responses [2].

In summary, there exists at the moment no consensus about the use of VEMPS in detecting a VS. So it seems currently not possible to make any diagnostic prognosis through VEMPS.

3.3. What Could Be the Most Probable Cause of Symptoms Like Hearing Loss or Vertigo in Patients with VS? Since it is not yet clear whether there are symptoms or parameters (such as tumour growth) which can be used to predict future hearing outcomes in VS patients, one might raise the question whether the hearing loss is caused by retrocochlear or cochlear mechanisms. Clinical and histological observations have suggested that the hearing loss in VS does not correlate with the size of the VS $[53,54]$, indicating that compression of the cochlear nerve within the internal auditory canal may not be the only mechanism in hearing loss. In other words, it may be that the commonly observed pure tone hearing loss patterns in patients with VS may be caused by cochlear, rather than exclusively retrocochlear mechanisms.

Roosli et al. performed a detailed assessment of cochlear pathology in patients with VS and also found a lack of correlation between tumour size and hearing loss. In a retrospective analysis of temporal bone histopathology they found that neural mechanisms caused by compression of the cochlear nerve are not solely responsible for hearing loss in patients with VS. When compared to the unaffected ear, VS caused significantly more inner and outer hair cell loss and cochlear neuronal loss, precipitate in endolymph and perilymph, and increased pure tone average thresholds. Moreover, the tumour distance from the cochlea and the nerve of origin did not correlate with structural changes in the cochlea or the hearing threshold. Direct invasion of the modiolus or the cochlea by VS was not observed in any case [55].

It is nowadays possible to demonstrate endolymphatic hydrops in vivo through MR imaging [56] and the degree of endolymphatic hydrops has been shown to correlate with loss of audiovestibular function in patients with Menière's disease [57-59]. MR imaging after local contrast application and image processing even enables the volumetric quantification of endolymphatic hydrops [60]. A classical picture of a VS on T1 weighted MRI after intravenous contrast application is shown in Figure 1. Figure 2 demonstrates vestibular endolymphatic hydrops in a patient with a stable (over 7 years) small 


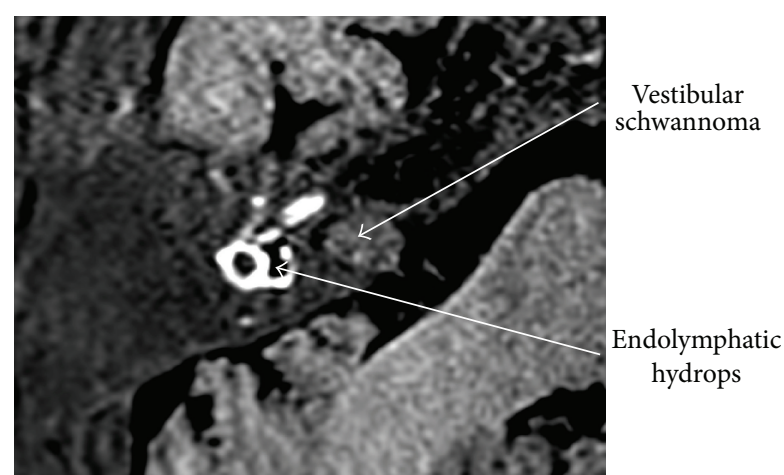

FIGURE 2: Endolymphatic hydrops and VS seen in a "locally enhanced inner ear MR imaging" (LEIM), 3D real inversion recovery MRI of the right inner ear after intratympanic Gadolinium contrast application. The vestibulum, the horizontal SCC and the basal turn of the cochlea are visible. Perilymph appears hyperintense, endolymph appears hypointense (black), and surrounding temporal bone appears grey. The vestibular endolymphatic space is clearly enlarged, indicating endolymphatic hydrops.

vestibular schwannoma who developed recurrent vertigo attacks during follow-up.

Naganawa et al. were able to identify vestibular endolymphatic spaces on noncontrast-enhanced 3D FLAIR (fluid attenuated inversion recovery) MRI. By using intrinsic perilymph signal increase in some patients with VS, endolymphatic hydrops was seen in four out of thirteen patients. The signal increase is attributed to the increased protein concentration [61]. In this small number of patients, there was no clear-cut correlation between symptoms and endolymphatic hydrops. However, the results suggest a surprisingly high prevalence of endolymphatic hydrops in VS patients and raise the possibility that, in analogy to Menière's disease, endolymphatic hydrops might contribute to audiovestibular function alterations in VS patients and this also raises the question whether specific therapeutic interventions directed against the endolymphatic hydrops might be useful, especially in patients during observational management of the VS.

It has been suggested that metabolic abnormalities caused by the tumour can have an important impact on the symptoms and that in this way abnormalities within the labyrinth may result in secondary injury to the organ of Corti. Thus, the progressive hearing loss could be caused by the release of toxins or potassium ions by the tumour. However, these metabolites have never been isolated or shown to be causative $[62,63]$.

The alteration of inner ear fluid composition in VS cases seems to play an important role. Different possible causes for this phenomenon have been described including impaired labyrinthine blood flow [64, 65], which may be induced, for example, by intralabyrinthine VS compression of the labyrinthine artery, cellular immune reactions [6668], and/or blockage of neuroaxonal transport mechanisms [69]. Moreover, acidophilic-staining precipitate in a series of 21 sporadic VS cases has been found [70].
Finally, a small subset of patients may experience a mixed hearing loss, due to an inner ear conductive loss thought to be caused by interference with the intracochlear perilymphatic fluid wave or dampening of stapes movement $[71,72]$.

The different causes of labyrinthine damage by a VS are summarized as follows:

Interference of microcirculation by nerve compression.

Release of toxins or potassium ions by the tumour.

Degeneration of the organ of Corti and the Stria vascularis.

Changes of inner ear homoeostasis.

Endolymphatic hydrops.

\section{Conclusion}

In order to preserve the auditory and vestibular function in VS patients, it is important not only to stop the growth of the tumour but also to avoid degenerative changes in the inner ear. A detailed neurotological workup helps in the diagnosis of VS of all sizes and can also provide useful prognostic information. In particular, caloric irrigation and unilateral hearing loss in audiometry have to draw the attention to the existence of a VS.

As false-negative results remain possible in small intrameatal tumours (1\%) [73] MR imaging is advisable even if the above tests are negative; in addition, MRI data is required in patients with positive tests so that an appropriate treatment plan (surgical intervention or watch and wait therapy) can be produced. It remains questionable whether an MRI scan is considered urgent when audiovestibular tests show no pathologies.

Loss of vestibular function usually occurs slowly and gradually, allowing an effective central compensation, so vertigo symptoms are often absent until the loss of function is severe.

Moreover, extended clinical vestibular function tests including vHIT and VEMPs can determine the overall extent of vestibular function loss, especially because the traditional caloric irrigation can deliver normal results in VS patients.

It has been shown that there is a significant degeneration of labyrinthine structures in ears with VS. Nevertheless, it is still unclear whether VS symptoms like hearing loss and vertigo are caused primarily by these labyrinthine mechanisms or by retrocochlear mechanisms.

Traditionally, VS have often been regarded simply as retrocochlear lesions. However, otoacoustic emission recordings and, in recent years, the endolymphatic hydrops visualization clearly show significant secondary labyrinthine pathology in patients with VS. Further research, using this new diagnostic possibility and correlating the morphologic findings with audiovestibular function test results in patients with VS will likely provide new insights into the relative contributions of neural versus labyrinthine factors to the pathophysiology and symptoms of VS. Some symptoms may possibly arise from labyrinthine factors rather than from neural factors. Since the inner ear is better accessible to therapeutic 
interventions than the audiovestibular nerve, for example, by intratympanic application of drugs, new therapeutic possibilities for symptom control in VS patients may possibly arise in cases where the tumour is stable and does not require invasive interventions.

\section{Competing Interests}

The authors declare that there is no conflict of interests regarding the publication of this paper.

\section{References}

[1] T. Tsutsumi, A. Tsunoda, Y. Noguchi, and A. Komatsuzaki, "Prediction of the nerves of origin of vestibular schwannomas with vestibular evoked myogenic potentials," The American Journal of Otology, vol. 21, no. 5, pp. 712-715, 2000.

[2] M. Ushio, S. Iwasaki, Y. Chihara et al., "Is the nerve origin of the vestibular schwannoma correlated with vestibular evoked myogenic potential, caloric test, and auditory brainstem response?" Acta Oto-Laryngologica, vol. 129, no. 10, pp. 1095-1100, 2009.

[3] R. Babu, R. Sharma, J. H. Bagley, J. Hatef, A. H. Friedman, and C. Adamsons, "Vestibular schwannomas in the modern era: epidemiology, treatment trends, and disparities in management," Journal of Neurosurgery, vol. 119, no. 1, pp. 121-130, 2013.

[4] D. B. Welling, M. E. Glasscock III, C. I. Woods, and C. G. Jackson, "Acoustic neuroma: a cost-effective approach," Otolaryngology_Head and Neck Surgery, vol. 103, no. 3, pp. 364-370, 1990.

[5] R. T. Sataloff, B. Davies, and D. L. Myers, "Acoustic neuromas presenting as sudden deafness," American Journal of Otology, vol. 6 , no. 4 , pp. 349-352, 1985.

[6] F. T. Shaia and J. L. Sheehy, "Sudden sensori-neural hearing impairment: a report of 1,220 cases," Laryngoscope, vol. 86, no. 3, pp. 389-398, 1976.

[7] M. Chaimoff, B. I. Nageris, J. Sulkes, T. Spitzer, and M. Kalmanowitz, "Sudden hearing loss as a presenting symptom of acoustic neuroma," American Journal of Otolaryngology-Head and Neck Medicine and Surgery, vol. 20, no. 3, pp. 157-160, 1999.

[8] D. Jethanamest, A. M. Rivera, H. Ji, V. Chokkalingam, F. F. Telischi, and S. I. Angeli, "Conservative management of vestibular schwannoma: predictors of growth and hearing," Laryngoscope, vol. 125, no. 9, pp. 2163-2168, 2015.

[9] J. N. Wagner, M. Glaser, B. Wowra et al., "Vestibular function and quality of life in vestibular schwannoma: does size matter?" Frontiers in Neurology, vol. 2, article 55, 2011.

[10] S.-E. Stangerup and P. Caye-Thomasen, "Epidemiology and natural history of vestibular schwannomas," Otolaryngologic Clinics of North America, vol. 45, no. 2, pp. 257-268, 2012.

[11] S.-E. Stangerup, J. Thomsen, M. Tos, and P. Cayé-Thomasen, "Long-term hearing preservation in vestibular schwannoma," Otology and Neurotology, vol. 31, no. 2, pp. 271-275, 2010.

[12] S.-E. Stangerup, P. Caye-Thomasen, M. Tos, and J. Thomsen, "Change in hearing during 'wait and scan' management of patients with vestibular schwannoma," Journal of Laryngology and Otology, vol. 122, no. 7, pp. 673-681, 2008.

[13] A.-S. Day, C.-T. Wang, C.-N. Chen, and Y.-H. Young, "Correlating the cochleovestibular deficits with tumor size of acoustic neuroma," Acta Oto-Laryngologica, vol. 128, no. 7, pp. 756-760, 2008 .
[14] K. M. Van Abel, M. L. Carlson, M. J. Link et al., "Primary inner ear schwannomas: a case series and systematic review of the literature," The Laryngoscope, vol. 123, no. 8, pp. 1957-1966, 2013.

[15] E. L. Slattery, S. C. Babu, R. A. Chole, and J. J. Zappia, "Intralabyrinthine schwannomas mimic cochleovestibular disease: symptoms from tumor mass effect in the labyrinth," Otology and Neurotology, vol. 36, no. 1, pp. 167-171, 2015.

[16] C. Jerin, E. Krause, B. Ertl-Wagner, and R. Gürkov, Clinical Features of Delayed Endolymphatic Hydrops and Intralabyrinthine Schwannoma, An Imaging-Confirmed Comparative Case Series, HNO, 2016.

[17] O. V. Tveiten, M. L. Carlson, F. Goplen, F. Vassbotn, M. J. Link, and M. Lund-Johansen, "Long-term auditory symptoms in patients with sporadic vestibular schwannoma: an international cross-sectional study," Neurosurgery, vol. 77, no. 2, pp. 218-227, 2015.

[18] R. A. Cueva, "Clinical thresholds for when to test for retrocochlear lesions: pro," Archives of Otolaryngology-Head and Neck Surgery, vol. 136, no. 7, pp. 725-727, 2010.

[19] A. B. Grayeli, M. Kalamarides, D. Bouccara, E. Ambert-Dahan, and O. Sterkers, "Auditory brainstem implant in neurofibromatosis type 2 and non-neurofibromatosis type 2 patients," Otology \& Neurotology, vol. 29, no. 8, pp. 1140-1146, 2008.

[20] M. J. Ruckenstein, R. A. Cueva, D. H. Morrison, and G. Press, "A prospective study of ABR and MRI in the screening for vestibular schwannomas," American Journal of Otology, vol. 17, no. 2, pp. 317-320, 1996.

[21] T. Murofushi and M. Takehisa, "Vestibular schwannoma with absent vestibular evoked myogenic potentials to clicks but normal ABR, caloric responses and vestibular evoked myogenic potentials to $500 \mathrm{~Hz}$ tone bursts," Acta Oto-Laryngologica, vol. 130 , no. 4, pp. 525-528, 2010.

[22] F. Telischi, "An objective method of analyzing cochlear versus noncochlear patterns of distortion-product otoacoustic emissions in patients with acoustic neuromas," Laryngoscope, vol. 110, no. 4, pp. 553-562, 2000.

[23] H. T. Gouveris, A. Victor, and W. J. Mann, "Cochlear origin of early hearing loss in vestibular schwannoma," Laryngoscope, vol. 117, no. 4, pp. 680-683, 2007.

[24] W. T. Koos, F. W. Böck, and R. F. Spetzler, Clinical Microneurosurgery, Thieme, Stuttgart, Germany, 1976.

[25] M. Samii and C. Matthies, "Management of 1000 vestibular schwannomas (acoustic neuromas): surgical management and results with an emphasis on complications and how to avoid them," Neurosurgery, vol. 40, no. 1, pp. 11-23, 1997.

[26] J. C. J. M. Artz, F. C. A. Timmer, J. J. S. Mulder, C. W. R. J. Cremers, and K. Graamans, "Predictors of future growth of sporadic vestibular schwannomas obtained by history and radiologic assessment of the tumor," European Archives of OtoRhino-Laryngology, vol. 266, no. 5, pp. 641-646, 2009.

[27] H. Borgmann, T. Lenarz, and M. Lenarz, "Preoperative prediction of vestibular schwannoma's nerve of origin with posturography and electronystagmography," Acta Oto-Laryngologica, vol. 131, no. 5, pp. 498-503, 2011.

[28] S. Tringali, A. Charpiot, M. Ould, C. Dubreuil, and C. FerberViart, "Characteristics of 629 vestibular schwannomas according to preoperative caloric responses," Otology and Neurotology, vol. 31, no. 3, pp. 467-472, 2010.

[29] M. Suzuki, C. Yamada, R. Inoue, A. Kashio, Y. Saito, and W. Nakanishi, "Analysis of vestibular testing in patients with 
vestibular schwannoma based on the nerve of origin, the localization, and the size of the tumor," Otology and Neurotology, vol. 29, no. 7, pp. 1027-1031, 2008.

[30] R. Teggi, A. Franzin, G. Spatola et al., "Vestibular assessment in patients with vestibular schwannomas: what really matters?" Acta Otorhinolaryngologica Italica, vol. 34, no. 2, pp. 123-128, 2014.

[31] K. R. Stidham and J. B. Roberson Jr., "Hearing improvement after middle fossa resection of vestibular schwannoma," Otology and Neurotology, vol. 22, no. 6, pp. 917-921, 2001.

[32] N. Perez and J. Rama-Lopez, "Head-impulse and caloric tests in patients with dizziness," Otology and Neurotology, vol. 24, no. 6, pp. 913-917, 2003.

[33] G. M. Halmagyi, I. S. Curthoys, P. D. Cremer et al., "The human horizontal vestibulo-ocular reflex in response to highacceleration stimulation before and after unilateral vestibular neurectomy," Experimental Brain Research, vol. 81, no. 3, pp. 479-490, 1990.

[34] A. Blödow, R. Helbig, N. Wichmann, M. Bloching, and L. E. Walther, "The video head impulse test: first clinical experiences," HNO, vol. 61, no. 4, pp. 327-334, 2013.

[35] A. Blodow, R. Helbig, N. Wichmann, A. Wenzel, L. E. Walther, and M. B. Bloching, "Video head impulse test or caloric irrigation? Contemporary diagnostic tests for vestibular schwannoma," HNO, vol. 61, no. 9, pp. 781-785, 2013.

[36] B. Machner, S. Gottschalk, T. Sander, C. Helmchen, and H. Rambold, "Intralabyrinthine schwannoma affecting the low but not high frequency function of the vestibulo-ocular reflex: Implications for the clinical diagnosis of chronic peripheral vestibular deficits," Journal of Neurology, Neurosurgery and Psychiatry, vol. 78, no. 7, pp. 772-774, 2007.

[37] M. Jorns-Häderli, D. Straumann, and A. Palla, "Accuracy of the bedside head impulse test in detecting vestibular hypofunction," Journal of Neurology, Neurosurgery and Psychiatry, vol. 78, no. 10, pp. 1113-1118, 2007.

[38] H. Scherer and A. H. Clarke, "The caloric vestibular reaction in space. Physiological considerations," Acta Oto-Laryngologica, vol. 100, no. 5-6, pp. 328-336, 1985.

[39] S. M. Rosengren, M. S. Welgampola, and J. G. Colebatch, "Vestibular evoked myogenic potentials: past, present and future," Clinical Neurophysiology, vol. 121, no. 5, pp. 636-651, 2010.

[40] C. Kantner and R. Gürkov, "Characteristics and clinical applications of ocular vestibular evoked myogenic potentials," Hearing Research, vol. 294, no. 1-2, pp. 55-63, 2012.

[41] E. Chiarovano, C. Darlington, P.-P. Vidal, G. Lamas, and C. De Waele, "The role of cervical and ocular vestibular evoked myogenic potentials in the assessment of patients with vestibular schwannomas," PLoS ONE, vol. 9, no. 8, Article ID e105026, 2014.

[42] M. S. Welgampola and J. G. Colebatch, "Characteristics and clinical applications of vestibular-evoked myogenic potentials," Neurology, vol. 64, no. 10, pp. 1682-1688, 2005.

[43] T. Murofushi, I. S. Curthoys, A. N. Topple, J. G. Colebatch, and G. M. Halmagyi, "Responses of guinea pig primary vestibular neurons to clicks," Experimental Brain Research, vol. 103, no. 1, pp. 174-178, 1995.

[44] T. Murofushi, G. M. Halmagyi, R. A. Yavor, and J. G. Colebatch, "Absent vestibular evoked myogenic potentials in vestibular neurolabyrinthitis. An indicator of inferior vestibular nerve involvement?" Archives of Otolaryngology-Head and Neck Surgery, vol. 122, no. 8, pp. 845-848, 1996.
[45] I. S. Curthoys, S. Iwasaki, Y. Chihara, M. Ushio, L. A. McGarvie, and A. M. Burgess, "The ocular vestibular-evoked myogenic potential to air-conducted sound; probable superior vestibular nerve origin," Clinical Neurophysiology, vol. 122, no. 3, pp. 611616, 2011.

[46] S. Iwasaki, T. Murofushi, Y. Chihara et al., "Ocular vestibular evoked myogenic potentials to bone-conducted vibration in vestibular schwannomas," Otology and Neurotology, vol. 31, no. 1, pp. 147-152, 2010.

[47] I. S. Curthoys, J. Kim, S. K. McPhedran, and A. J. Camp, "Bone conducted vibration selectively activates irregular primary otolithic vestibular neurons in the guinea pig," Experimental Brain Research, vol. 175, no. 2, pp. 256-267, 2006.

[48] M. Kinoshita, S. Iwasaki, C. Fujimoto et al., "Ocular vestibular evoked myogenic potentials in response to air-conducted sound and bone-conducted vibration in vestibular schwannoma," Otology and Neurotology, vol. 34, no. 7, pp. 1342-1348, 2013.

[49] T. Murofushi, M. Matsuzaki, and M. Mizuno, "Vestibular evoked myogenic potentials in patients with acoustic neuromas," Archives of Otolaryngology-Head and Neck Surgery, vol. 124, no. 5, pp. 509-512, 1998.

[50] T. Patko, P.-P. Vidal, N. Vibert, P. Tran Ba Huy, and C. De Waele, "Vestibular evoked myogenic potentials in patients suffering from an unilateral acoustic neuroma: a study of 170 patients," Clinical Neurophysiology, vol. 114, no. 7, pp. 1344-1350, 2003.

[51] M. Ushio, M. Matsuzaki, H. Takegoshi, and T. Murofushi, "Click- and short tone burst-evoked myogenic potentials in cerebellopontine angle tumors," Acta Oto-Laryngologica. Supplementum, no. 545, pp. 133-135, 2001.

[52] Y.-F. Hu, P.-W. Cheng, and Y.-H. Young, "Comparison of vestibular function between large cerebellopontine angle meningioma and schwannoma," Acta Oto-Laryngologica, vol. 129, no. 2, pp. 161-165, 2009.

[53] M. A. Arriaga, S. Long, and R. Nelson, "Clinical correlates of acoustic neuroma volume," Otology and Neurotology, vol. 14, no. 5, pp. 465-468, 1993.

[54] J. B. Nadol Jr., P. F. Diamond, and A. R. Thornton, "Correlation of hearing loss and radiologic dimensions of vestibular schwannomas (acoustic neuromas)," American Journal of Otology, vol. 17, no. 2, pp. 312-316, 1996.

[55] C. Roosli, F. H. Linthicum, S. Cureoglu, and S. N. Merchant, "Dysfunction of the cochlea contributing to hearing loss in acoustic neuromas: an underappreciated entity," Otology and Neurotology, vol. 33, no. 3, pp. 473-480, 2012.

[56] C. Jerin, E. Krause, B. Ertl-Wagner, and R. Gürkov, "Longitudinal assessment of endolymphatic hydrops with contrastenhanced magnetic resonance imaging of the labyrinth," Otology and Neurotology, vol. 35, no. 5, pp. 880-883, 2014.

[57] R. Gürkov, W. Flatz, J. Louza, M. Strupp, and E. Krause, "In vivo visualization of endolyphatic hydrops in patients with Meniere's disease: correlation with audiovestibular function," European Archives of Oto-Rhino-Laryngology, vol. 268, no. 12, pp. 17431748, 2011.

[58] R. Gürkov, W. Flatz, J. Louza, M. Strupp, B. Ertl-Wagner, and E. Krause, "Herniation of the membranous labyrinth into the horizontal semicircular canal is correlated with impaired caloric response in Ménière's disease," Otology and Neurotology, vol. 33, no. 8, pp. 1375-1379, 2012.

[59] R. Gürkov, W. Flatz, J. Louza, M. Strupp, B. Ertl-Wagner, and E. Krause, "In vivo visualized endolymphatic hydrops and inner ear functions in patients with electrocochleographically 
confirmed Ménière's disease," Otology \& Neurotology, vol. 33, no. 6, pp. 1040-1045, 2012.

[60] R. Gürkov, A. Berman, O. Dietrich et al., "MR volumetric assessment of endolymphatic hydrops," European Radiology, vol. 25, no. 2, pp. 585-595, 2015.

[61] S. Naganawa, H. Kawai, M. Sone, T. Nakashima, and M. Ikeda, "Endolympathic hydrops in patients with vestibular schwannoma: visualization by non-contrast-enhanced 3D FLAIR," Neuroradiology, vol. 53, no. 12, pp. 1009-1015, 2011.

[62] H. L. DeLozier, R. R. Gacek, and S. T. Dana, "Intralabyrinthine schwannoma," Annals of Otology, Rhinology and Laryngology, vol. 88, no. 2, part 1, pp. 187-191, 1979.

[63] R. T. Miyamoto, S. F. Isenberg, W. M. Culp, and L. B. Tubergen, "Isolated intralabyrinthine schwannoma," American Journal of Otology, vol. 1, no. 4, pp. 215-217, 1980.

[64] T. Nakashima, S. Naganawa, M. Sone et al., "Disorders of cochlear blood flow," Brain Research Reviews, vol. 43, no. 1, pp. 17-28, 2003.

[65] T. Matsunaga and J. Kanzaki, "Morphological evidence that impaired intraneural microcirculation is a possible mechanism of eighth nerve conduction block in acoustic neuromas," European Archives of Oto-Rhino-Laryngology, vol. 257, no. 8, pp. 412417, 2000.

[66] N. Rasmussen, K. Bendtzen, J. Thomsen, and M. Tos, "Patientspecific cell-mediated immunity against human acoustic neuroma extract," Acta Oto-Laryngologica, vol. 94, no. 3-4, pp. 261265, 1982.

[67] N. Rasmussen, K. Bendtzen, J. Thomsen, and M. Tos, "Specific cellular immunity in acoustic neuroma patients," Otolaryngology-Head and Neck Surgery, vol. 91, no. 5, pp. 532-536, 1983.

[68] N. Rasmussen, K. Bendtzen, J. Thomsen, and M. Tos, "Antigenicity and protein content of perilymph in acoustic neuroma patients," Acta Oto-Laryngologica, vol. 97, no. 5-6, pp. 502-508, 1984.

[69] J. Thomsen, O. Saxtrup, and M. Tos, "Quantitated determination of proteins in perilymph in patients with acoustic neuromas," ORL: Journal for Oto-Rhino-Laryngology and Its Related Specialties, vol. 44, no. 2, pp. 61-65, 1982.

[70] L. Eckermeier, W. Pirsig, and D. Mueller, "Histopathology of 30 non-operated acoustic schwannomas," Archives of Oto-RhinoLaryngology, vol. 222, no. 1, pp. 1-9, 1979.

[71] R. J. Kennedy, C. Shelton, K. L. Salzman, H. C. Davidson, and H. R. Harnsberger, "Intralabyrinthine schwannomas: diagnosis, management, and a new classification system," Otology and Neurotology, vol. 25, no. 2, pp. 160-167, 2004.

[72] M. S. Karlan, M. Basek, and G. B. Potter, "Intracochlear neurilemmoma," Archives of Otolaryngology, vol. 96, no. 6, pp. 573-575, 1972.

[73] B. K. Diallo, V. Franco-Vidal, D. Vasili et al., "The neurotologic evaluation of vestibular schwannomas. Results of audiological and vestibular testing in 100 consecutive cases," Revue de Laryngologie Otologie Rhinologie, vol. 127, no. 4, pp. 203-209, 2006. 


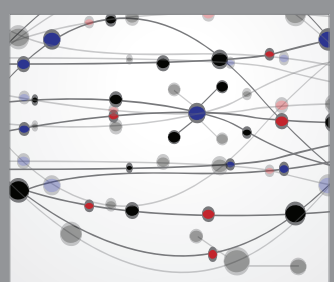

The Scientific World Journal
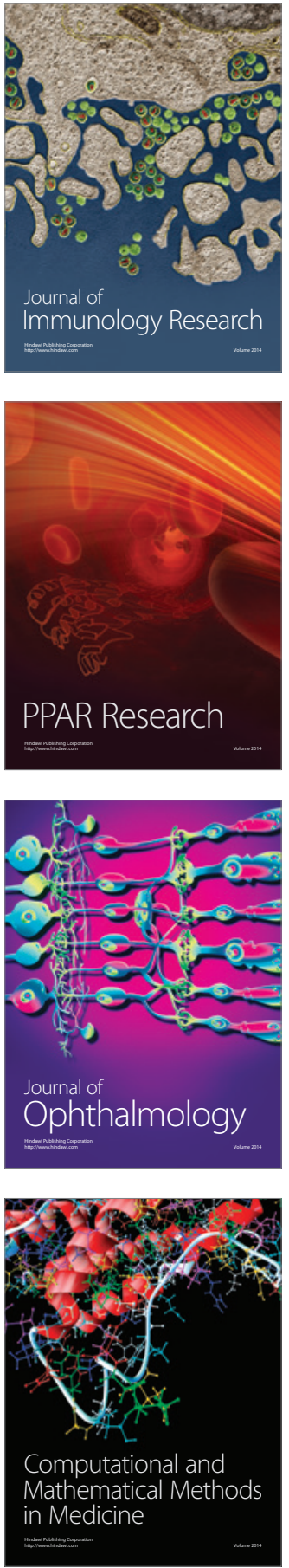

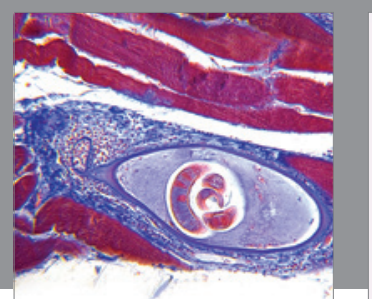

Gastroenterology Research and Practice

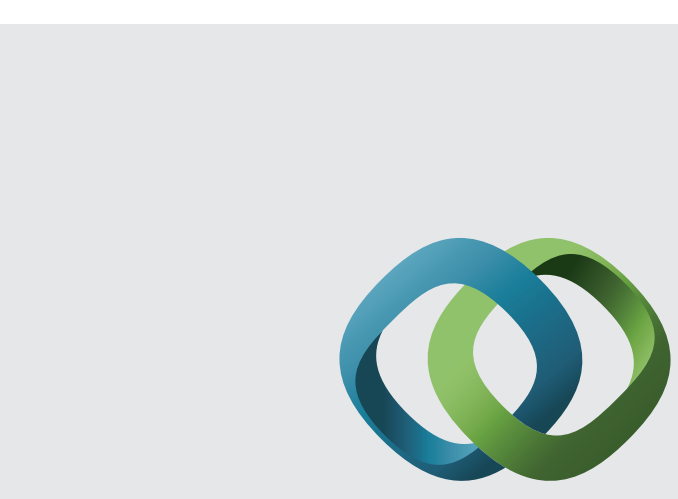

\section{Hindawi}

Submit your manuscripts at

http://www.hindawi.com
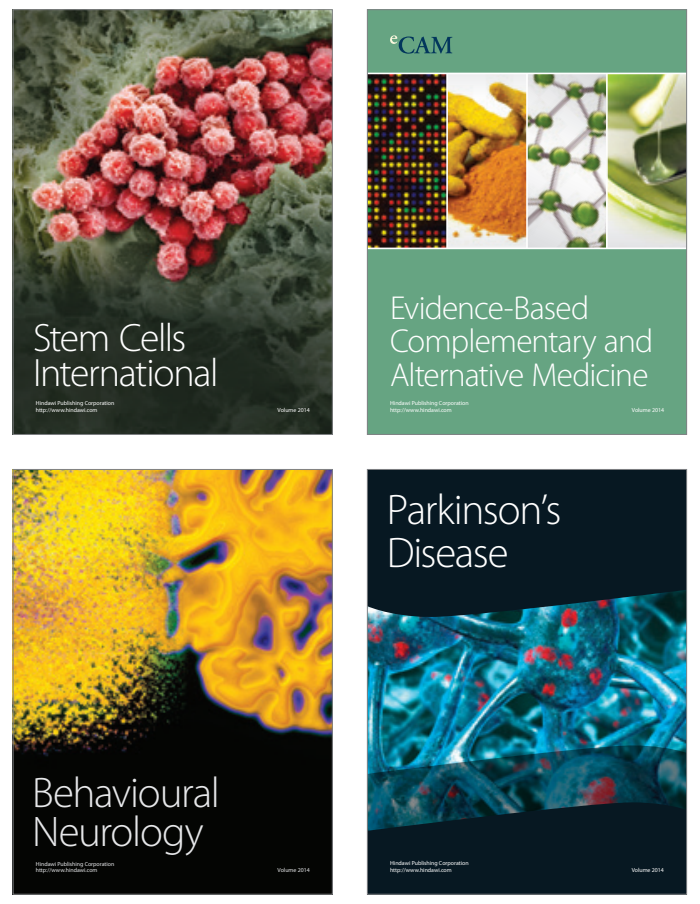
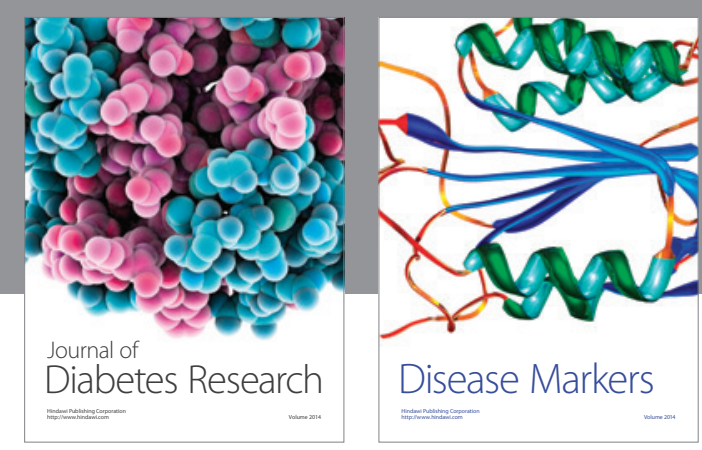

Disease Markers
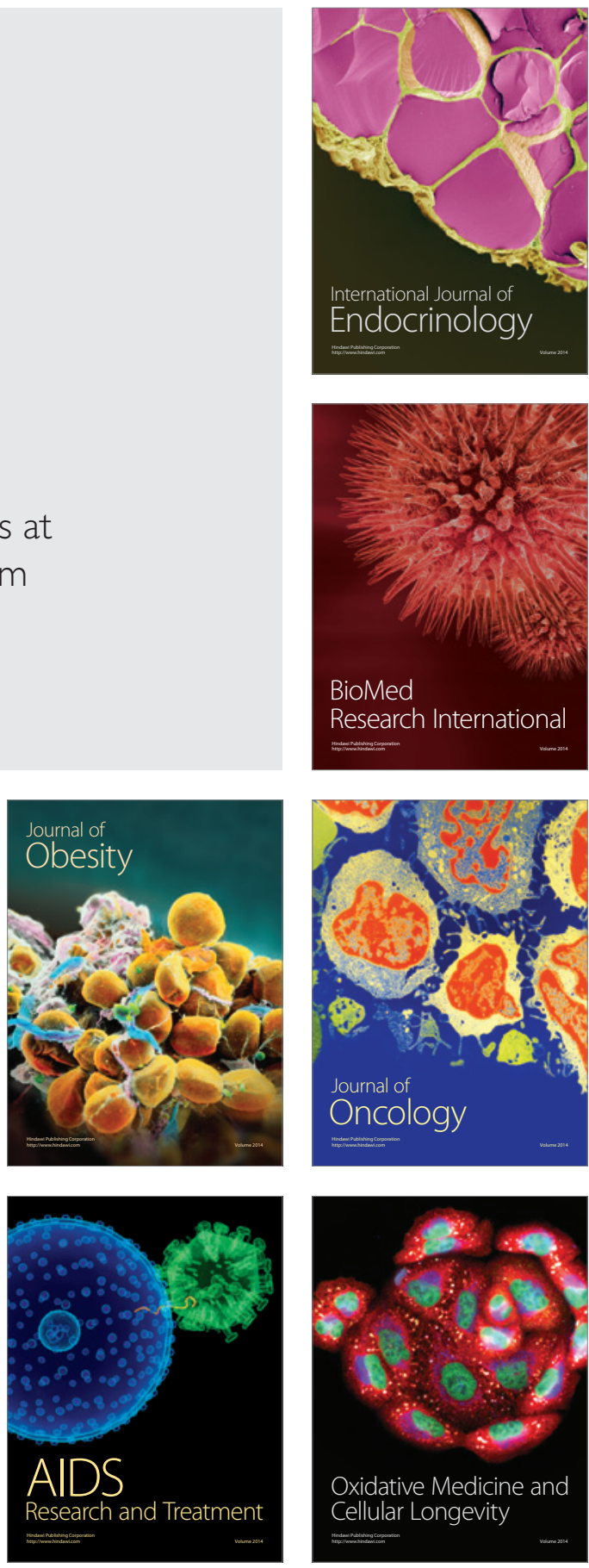